\title{
Correlation between live weight and body measurements in certain dog breeds
}

\author{
B. Yüceer Özkul ${ }^{1 \#}$, P.C.K. Doka², D. Özen ${ }^{3}$, F.T. Özbaşer ${ }^{4}$, B. Özarslan ${ }^{5}$ \& \\ F. Atasoy ${ }^{1}$ \\ ${ }^{1}$ Ankara University, Faculty of Veterinary Medicine, Department of Animal Husbandry, Ankara, Turkey \\ ${ }^{2}$ AKD, Kids, Ankara, Turkey \\ ${ }^{3}$ Ankara University, Faculty of Veterinary Medicine, Department of Biostatistics, Ankara, Turkey \\ ${ }^{4}$ Namık Kemal University, Faculty of Veterinary Medicine, Department of Animal Husbandry, Tekirdağ, Turkey \\ ${ }^{5}$ Kırıkkale University, Faculty of Veterinary Medicine, Department of Animal Husbandry, Kırıkkale, Turkey
}

(Submitted 18 November 2020; Accepted 15 January 2021; Published 15 February 2021)
Copyright resides with the authors in terms of the Creative Commons Attribution 4.0 South African License.
See: http://creativecommons.org/licenses/by/4.0/za
Condition of use: The user may copy, distribute, transmit and adapt the work, but must recognize the authors and the South African Journal of Animal Science.

\begin{abstract}
The purpose of this study was to determine the correlation between live weight and body measurements in Zağar, Zerdava, and Çatalburun dogs. Animal materials were obtained from various regions of Turkey. A total of 304 dogs from three breeds were used: Zağar (45 females, 59 males), Zerdava (50 females, 50 males), and Çatalburun (62 females, 38 males). Live weights and certain body measurements were determined. A linear regression model was created using the parameters obtained in this study. The bodyweights calculated with the body measurements were found to be at a high or acceptable level in the Zağar, Zerdava, and Çatalburun genotypes $\left(R^{2}=0.902,0.467\right.$, and 0.697 , respectively).
\end{abstract}

Keywords: body measurements, breed, correlation, dog, live weight

\# Corresponding author: yuceerbanu@hotmail.com

\section{Introduction}

Turkey is rich in animal genetic resources. As a result of the various geographical structures and the large surface area there are many genetic types of dog in Turkey. They have been bred to maintain breed purity in Anatolia for centuries and are adapted to the conditions of the region. Most of these dogs are used to herd sheep or to hunt. The Zağar, Zerdava, and Çatalburun breeds are hunting dogs (Atasoy, 2009). Zağar dogs (Figure 1A) are raised mainly in the mountainous regions of Turkey, where a nomadic culture is dominant, and are used for rabbit hunting in particular. Zerdava dogs (Figure 1B) are bred in Trabzon and other parts of the Black Sea region. This dog is the only Turkish native breed with upright ears, and is used primarily as a guard dog and for boar hunting (Atasoy, 2009). Çatalburun dogs (Figure 1C) are bred in Mersin. In addition to being preferred as hunting dogs, they have recently been used to detect narcotics (Atasoy, 2009; Petek \& Dinçel, 2012).

The bodyweights of dogs influence their usefulness for breeding, racing and as watch dogs. Çelik \& Yilmaz (2018) found withers height, abdominal width, rump height and chest depth were predictive of the bodyweight of Turkish Tazi dogs. Thus, the aim of this study was to develop equations that could be used to predict the bodyweight of Zağar, Zerdava, and Çatalburun dogs, using linear measures of the dimensions of their bodies. 

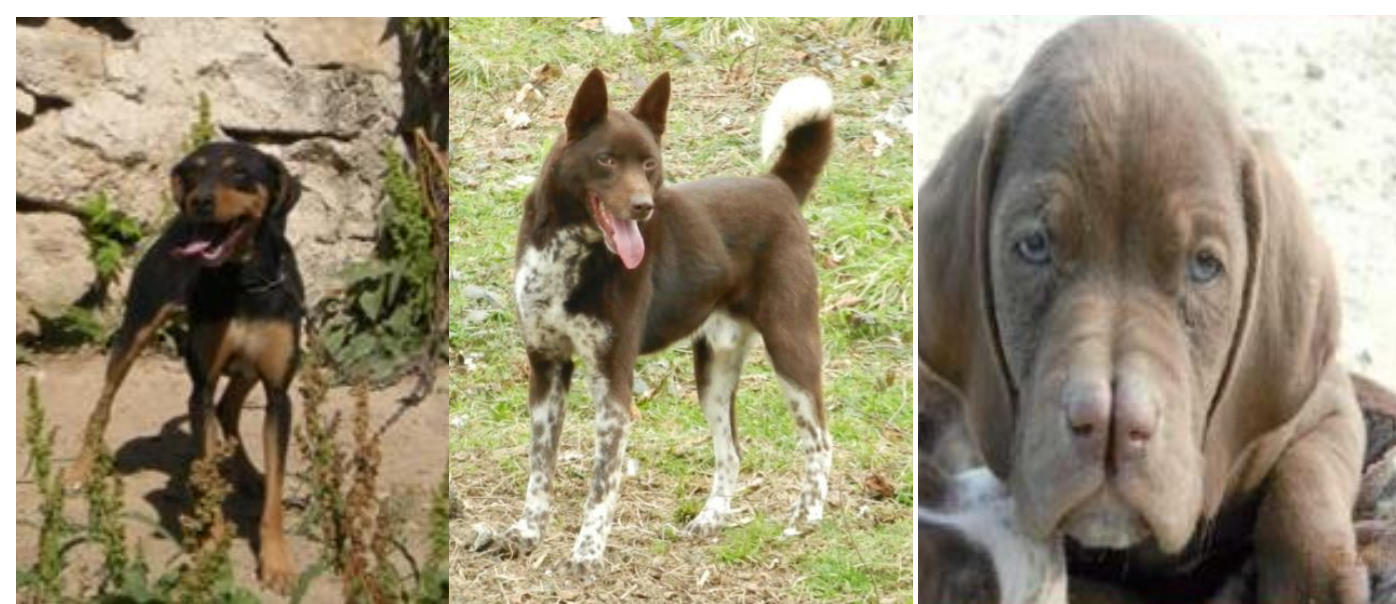

Figure 1 Examples of the Zağar Zerdava, and Çatalburun breeds

\section{Materials and Methods}

This study was carried out after obtaining ethical approval (No. 2013-18-135; dated 23/10/2013) from Ankara University Animal Experiments Local Ethics Board. The data originated from Zağar $(n=104 ; 45$ females, 59 males), Zerdava ( $n=100 ; 50$ females, 50 males), and Çatalburun ( $n=100 ; 62$ females, 38 males) dogs that were at least 12 months old and bred in various regions of Turkey. Data were obtained from Project No: 1150 613, supported by The Scientific and Technological Research Council of Turkey. The dogs were weighed on a scale with $100 \mathrm{~g}$ precision. Withers height, rump height, body length, chest width, and chest depth were measured with a ruler. Chest circumference, front and hind cannon bone circumference, head, face, ear, and tail length, ear width, ears and eyes interval, mouth circumference and tail width were measured with a measuring tape (Spira, 1991; Tepeli \& Çetin, 2000; Tepeli \& Çetin, 2003).

Stata 14 (StataCorp, LLC, College Station, Texas, USA) was used for the statistical analyses. Pearson correlation coefficients were calculated to determine the strength and direction of the relationship between pairs of variables. Backward stepwise multiple linear regression was used to estimate live weights of the dogs from the measurements that describe each of the breeds. Tolerance and the variance inflation factor were used to assess collinearity among the predictor variables. Variables entered the model with probability $P=0.05$ and exited at probability $P=0.10$. Durbin-Watson statistics were used to examine the presence of autocorrelation.

\section{Results and Discussion}

The dogs that provided the data used in this study were described by their weight, age and 17 linear measures (Table 1). A portion of these data were reported in Özbașer et al. (2020) and have been provided here to describe the origins of the data used in this study more completely.

The estimated correlation coefficients between the body measurements of Zağar, Zerdava, and Çatalburun dogs are shown in Appendix Tables 1,2 and 3. There have been no previous scientific studies of the relationships between bodyweight and dimensions using samples of these breeds of dog. The correlations among the body dimensions and with weight were generally positive and significantly greater than zero. For all three breeds, the relatively large and highly significant correlations among the body dimensions led to the anticipation of potential issues with collinearity among the independent variables that were to be used in developing the prediction equations. For the Zağar dogs, the correlations of most of the body dimensions with age were not significantly different from zero, indicating that these dogs were essentially full grown when the data were recorded. However, many of the correlations between body dimensions and age were significant for the Zerdava and Çatalburun dogs, which may still have been growing when the data were recorded. This observation is consistent with the difference in the average age of the dogs from which data were recorded.

For the Zağar dogs, the regression analysis resulted in a prediction equation for bodyweight that was based on five independent variables (Table 2). It was concluded that live weight could be adequately predicted from body length, chest width, chest circumference, behind cannon bone circumference, and ears interval. This equation explained $90.2 \%$ of the total variance in the live weight. The tolerance statistics and variance inflation factors for this final model suggest there was not a problem with multi-collinearity. 
Table 1 Estimates of the mean and standard deviation describing Zağar, Zerdava, and Çatalburun dogs that were used in this study

\begin{tabular}{lrrrrrr}
\hline & \multicolumn{2}{c}{ Zağar dogs } & \multicolumn{2}{c}{ Zerdava dogs } & \multicolumn{2}{c}{ Çatalburun dogs } \\
\cline { 5 - 7 } Measurement & Mean & SD & Mean & SD & Mean & SD \\
\hline & 18.24 & 3.83 & 15.96 & 3.64 & 19.9 & 4.09 \\
Bodyweight, kg & 3.12 & 1.02 & 2.08 & 1.62 & 2.12 & 1.76 \\
Age, years & 50.51 & 4.03 & 48.07 & 2.54 & 47.8 & 3.65 \\
Withers height, cm & 50.28 & 4.05 & 47.11 & 2.86 & 48.68 & 3.51 \\
Rump height, cm & 56.42 & 3.97 & 52.28 & 3.16 & 53.80 & 3.34 \\
Body length, cm & 17.78 & 1.68 & 17.56 & 1.83 & 19.24 & 1.9 \\
Chest width, cm & 21.01 & 2.05 & 20.36 & 1.56 & 22.21 & 1.7 \\
Chest depth, cm & 58.11 & 4.89 & 53.35 & 3.94 & 59.07 & 4.26 \\
Chest circumference, cm & 9.83 & 0.86 & 9.04 & 0.58 & 9.18 & 0.75 \\
Circumference front cannon bone, cm & 8.97 & 0.85 & 8.54 & 0.64 & 8.51 & 0.58 \\
Circumference hind cannon bone, cm & 23.58 & 1.50 & 20.97 & 1.06 & 19.87 & 1.71 \\
Head length, cm & 9.67 & 0.85 & 8.48 & 0.56 & 7.25 & 0.83 \\
Face length, cm & 14.26 & 1.30 & 10.03 & 0.76 & 14.7 & 1.69 \\
Ear length, cm & 10.96 & 1.17 & 8.73 & 0.63 & 11.68 & 0.85 \\
Ear width, cm & 13.39 & 1.26 & 10.80 & 1.58 & 13.68 & 1.04 \\
Distance between ears, cm & 4.52 & 0.60 & 4.55 & 1.56 & 4.90 & 0.37 \\
Distance between eyes, cm & 20.75 & 2.01 & 18.13 & 1.19 & 18.91 & 1.44 \\
Mouth circumference, cm & 35.43 & 3.48 & 28.97 & 2.96 & & \\
Tail length, cm & & & 6.88 & 2.77 & & \\
Tail width, cm & & & & & &
\end{tabular}

Table 2 Final regression model to predict bodyweight in Zağar dogs from their body dimensions

\begin{tabular}{lccccc}
\hline Independent variable & $\mathrm{b} \pm \mathrm{SE}$ & $P$-value & Bounds of $95 \% \mathrm{Cl}$ & Tolerance & VIF \\
\hline Intercept & $-30.446 \pm 1.879$ & $<0.001$ & $-34.175<b<-26.716$ & & \\
Body length & $0.148 \pm 0.048$ & 0.002 & $0.054<b<0.243$ & 0.409 & 2.443 \\
Chest width & $0.285 \pm 0.126$ & 0.026 & $0.035<b<0.534$ & 0.330 & 3.032 \\
Chest circumference & $0.432 \pm 0.047$ & $<0.001$ & $0.338<b<0.526$ & 0.273 & 3.660 \\
Hind cannon bone circumference & $0.821 \pm 0.246$ & 0.001 & $0.333<b<1.308$ & 0.335 & 2.984 \\
Ear interval & $0.208 \pm 0.110$ & 0.062 & $-0.010<b<0.427$ & 0.766 & 1.306 \\
\hline
\end{tabular}

b: regression coefficient, Cl: confidence interval, VIF: variance inflation factor

Descriptive statistics that characterize the distribution of predicted live weight of Zağar dogs in comparison with their observed live weights are presented in Table 3. As expected, the means of the predicted and observed values were identical. The distribution of the predicted values was trimmed only slightly compared with the observed values. 
Table 3 Correspondence of observed and predicted live weights of Zağar dogs

\begin{tabular}{lcccccc}
\hline & $\begin{array}{c}\text { Arithmetic } \\
\text { mean }\end{array}$ & SE & $\begin{array}{c}\text { Standard } \\
\text { deviation }\end{array}$ & Median & Minimum & Maximum \\
\hline Observed live weight & 18.24 & 0.38 & 3.83 & 17.85 & 9.80 & 28.00 \\
Predicted live weight & 18.24 & 0.36 & 3.64 & 17.91 & 9.86 & 26.85 \\
\hline
\end{tabular}

For the Zerdava dogs, the regression analysis resulted in a prediction equation for bodyweight that was based on four independent variables (Table 4). It was concluded that live weight could be predicted from wither height, chest width, chest circumference, and head length. This equation explained $47.5 \%$ of the total variance in the live weight. The tolerance statistics and variance inflation factors for this final model suggest there was not a problem with multi-collinearity.

Table 4 Final regression model to predict body weight in Zerdava dogs from their body dimensions

\begin{tabular}{lrcccc}
\hline Independent variable & $\mathrm{b} \pm \mathrm{SE}$ & $P$-value & Bounds of $95 \% \mathrm{Cl}$ & Tolerance & VIF \\
\hline Intercept & $-33.219 \pm 6.182$ & $<0.001$ & $-45.495<\mathrm{b}<-20.943$ & & \\
Wither height & $0.304 \pm 0.141$ & 0.034 & $0.023<\mathrm{b}<0.584$ & 0.577 & 1.733 \\
Chest width & $0.547 \pm 0.165$ & 0.001 & $0.219<\mathrm{b}<0.875$ & 0.821 & 1.219 \\
Chest circumference & $0.251 \pm 0.093$ & 0.008 & $0.066<b<0.435$ & 0.563 & 1.775 \\
Head length & $0.554 \pm 0.334$ & 0.090 & $-0.109<b<1.217$ & 0.611 & 1.636 \\
& & & & & \\
\hline
\end{tabular}

Descriptive statistics characterizing the distribution of predicted live weight of Zerdava dogs in comparison with their observed live weights are presented in Table 5. As expected, the means of the predicted and observed values were identical, as they were for the Zağar. However, as indicated by the lower $R^{2}$ value for this equation, the predicted variables were substantially less variable than the observations and this was particularly notable for the heavier dogs.

Table 5 Correspondence of observed and predicted live weights of Zerdava dogs

\begin{tabular}{lcccccc}
\hline & $\begin{array}{c}\text { Arithmetic } \\
\text { mean }\end{array}$ & SE & $\begin{array}{c}\text { Standard } \\
\text { deviation }\end{array}$ & Median & Minimum & Maximum \\
\hline Observed live weight & 15.96 & 0.36 & 3.64 & 15.00 & 10.00 & 29.50 \\
Predicted live weight & 15.96 & 0.25 & 2.51 & 16.01 & 11.16 & 24.32 \\
\hline
\end{tabular}

For the Çatalburun dogs, the regression analysis resulted in a prediction equation for bodyweight that was based on three independent variables (Table 6). It was concluded that live weight could be predicted from withers height, body length, and chest circumference. This equation explained $69.7 \%$ of the total variance in the live weight. The tolerance statistics and variance inflation factors for this final model suggested there was not a problem with multi-collinearity. 
Table 6 Final regression model to predict bodyweight in Çatalburun dogs from their body dimensions

\begin{tabular}{lccccc}
\hline Independent variable & $\mathrm{b} \pm \mathrm{SE}$ & $P$-value & Bounds of $95 \% \mathrm{Cl}$ & Tolerance & VIF \\
\hline Intercept & $-23.73 \pm 3.917$ & $<0.001$ & $-31.512<b<-15.962$ & & \\
Withers height & $0.194 \pm 0.096$ & 0.046 & $0.003<b<0.384$ & 0.432 & 2.317 \\
Body length & $-0.203 \pm 0.091$ & 0.029 & $-0.384<b<-0.021$ & 0.568 & 1.760 \\
Chest circumference & $0.767 \pm 0.081$ & $<0.001$ & $0.605<b<0.928$ & 0.443 & 2.259 \\
\hline
\end{tabular}

Descriptive statistics characterizing the distribution of predicted live weight of Çatalburun dogs in comparison with their observed live weights are presented in Table 7 . As indicated by the $R^{2}$ value for this equation being intermediate between the high value that for Zağar dogs and the lower value for Zerdava dogs, the predicted variables were less variable than the observations, but not so much as for the Zerdava dogs.

Table 7 Correspondence of observed and predicted live weights of Çatalburun dogs

\begin{tabular}{lcccccc}
\hline & $\begin{array}{c}\text { Arithmetic } \\
\text { mean }\end{array}$ & SE & $\begin{array}{c}\text { Standard } \\
\text { deviation }\end{array}$ & Median & Minimum & Maximum \\
\hline Observed live weight & 19.90 & 0.41 & 4.09 & 19.05 & 12.50 & 28.60 \\
Predicted live weight & 19.90 & 0.34 & 3.42 & 19.67 & 13.83 & 29.20 \\
\hline
\end{tabular}

The prediction equations were specific to the breed of dog, although chest circumference was included as a dependent variable in all of the equations. The correlation coefficients of chest circumference with body weight were quite high in the Zağar $(r=0.914)$ and Çatalburun $(r=0.822)$ breeds, but much lower in the Zerdava $(r=0.232)$. Whether these equations were adequate would depend on the accuracy of prediction that was needed in any particular application.

In this study, significant positive correlations were found between live weight and body measurements. Similar results were obtained by Dirlik (2008) for the German Shepherd, Labrador Retrievers, and Belgian Malinois breeds and by Emehelu et al. (2012) for Nigerian native dogs. In their study, chest circumference had the highest correlation with bodyweight among the morphometric measurements. However, unlike the present study, ear length was found to be a significant predictor of live weight in a study conducted on German Shepherd dogs by the Gemlik Military Veterinary School (Elmaz et al., 2006).

Çelik and Yilmazn (2018) explained $68 \%$ and $91 \%$ of the variation in live weight bodyweight of Turkish Tazi dogs with morphometric measures using the classification and regression tree method and multivariate adaptive regression splines, respectively. It is noteworthy that the less computationally demanding methods used in this study achieved similar accuracy in prediction of live weight.

\section{Conclusion}

The measured live weights of the Zağar, Zerdava, and Çatalburun genotypes and predictions of live weight from morphometric measures indicate that the weight of these dogs can be estimated with reasonable accuracy. Thus, linear measures of the body dimensions of these dogs may be indicative of their usefulness in breeding, racing, and keeping watch.

\section{Acknowledgements}

The authors would like to thank Scientific and Technological Research Council of Turkey (TUBITAK) and the owners of the Zağar, Zerdava and Çatalburun dogs for their support to the project.

\section{Authors' Contributions}

This article was produced from PCKD's master's thesis completed under the supervision of BYO. FA, BYO, FTO, and BO contributed to the concept, design and execution of the study. PCKD and DO conducted the statistical analyses. BYO supervised the experiment and wrote the manuscript. 


\section{Conflict of Interest Declaration}

The authors declare there are no competing interests.

\section{References}

Atasoy, F., 2009. Kedi - köpek ırkları ve yetiştiriciliği ders notları. Ankara Üniversitesi, Veteriner Fakültesi, Zootekni Anabilim Dalı, Ankara.

Çelik, S. \& Yilmaz, O., 2018. Prediction of body weight of Turkish Tazi dogs using data mining techniques: Classification and regression tree (CART) and multivariate adaptive regression splines (MARS). Pakistan J. Zool. 50(2), 575583. DOI: 10.17582/journal.pjz/2018.50.2.575.583

Dirlik, H., 2008. Gemlik askeri veteriner okulu ve eğitim merkezi komutanlığında yetiştirilen bazı köpek ırklarında vücut ölçüleri ve bu ölçüler arasındaki fenotipik korelasyonlar. Adnan Menderes Üniversitesi Sağlık Bilimleri Enstitüsü Yüksek Lisans Tezi.

Elmaz, O., Aksoy, O.A., Dikmen, S. \& Zonturlu, A., 2006. Growth performance, survival ratio and body measurements until weaning age of German Shepherd dog. World Small Animal Veterinary Association, Proc. 31st World Congress, Prague, Czech Republic.

Emehelu, C.O., Eze, J.I., Akune, A. \& Chah, K.F., 2012. Estimation of live body weight from body measurements in Nigerian local dogs. Tropical Veterinarian 30, 65-73.

Petek, M. \& Dinçel, D., 2012. Çatalburun köpekleri ve ırk özellikleri. Uludağ Üniv. J. Fac.Vet. Med. 1, 25 - 28.

Özbașer, F.T., Atasoy, F., Erdoğan, M., Yüceer Özkul, B. \& Özarslan. B., 2020. Morphological and genetic characteristics of Zerdava, a native Turkish dog breed. Kafkas Univ. Vet. Fak. Derg. 26(5), 617-623, DOI: 10.9775/kvfd.2020.24004

Spira, R.H., 1991. Canine terminology. Howell Book House, New York, USA.

Tepeli, C. \& Çetin, O., 2000. Kangal ırkı Türk çoban köpeklerinde büyüme, bazı vücut ölçüleri ve döl verimi özelliklerinin belirlenmesi. Vet. Bil. Derg. 16 (1), 5-16.

Tepeli, C. \& Çetin, O., 2003. A study on head measurements of Kangal and Akbas Turkish shepherd dogs. The Journal of Veterinary Science 19 (1-2), 17-20. 
Appendix Table 1 Estimates of phenotypic correlations among age and body dimensions in Zağar dogs

\begin{tabular}{|c|c|c|c|c|c|c|c|c|c|c|c|c|c|c|c|c|c|}
\hline & Age & WH & $\mathrm{RH}$ & $B L$ & $\mathrm{CW}$ & $C D$ & $\mathrm{CC}$ & FC & $\mathrm{BC}$ & $\mathrm{HL}$ & $\mathrm{FL}$ & EL & $\mathrm{EW}$ & EAl & EYI & MC & TL \\
\hline Age & 1 & 0.089 & 0.130 & 0.072 & 0.131 & 0.186 & $0.198^{*}$ & 0.010 & 0.038 & -0.001 & 0.007 & -0.063 & -0.018 & 0.094 & 0.115 & 0.061 & -0.041 \\
\hline WH & & 1 & $0956^{\star \star}$ & $0.713^{\star *}$ & $0.453^{\star *}$ & $0.575^{\star *}$ & $0.651^{* *}$ & $0.565^{\star \star}$ & $0.685^{\star *}$ & $0.650^{* *}$ & $0.631^{* *}$ & $0.553^{\star *}$ & $0.483^{\star *}$ & $0.445^{\star \star}$ & $0.391^{* *}$ & $0.481^{* *}$ & $0.512^{\star *}$ \\
\hline $\mathrm{RH}$ & & & 1 & $0.728^{\star *}$ & $0.493^{\star *}$ & $0.600^{* *}$ & $0.670^{* *}$ & $0.587^{\star *}$ & $0.710^{\star *}$ & $0.621^{* *}$ & $0.623^{\star *}$ & $0.541^{\star *}$ & $0.496^{\star *}$ & $0.484^{\star \star}$ & $0.449^{* *}$ & $0.522^{\star *}$ & $0.517^{\star \star}$ \\
\hline $\mathrm{BL}$ & & & & 1 & $0.568^{\star *}$ & $0.583^{\star *}$ & $0.663^{* *}$ & $0.730^{* *}$ & $0.740^{\star *}$ & $0.581^{* *}$ & $0.537^{\star *}$ & $0.594^{\star *}$ & $0.630^{\star *}$ & $0.430^{* *}$ & $0.548^{* *}$ & $0.615^{\star *}$ & $0.517^{\star \star}$ \\
\hline $\mathrm{CW}$ & & & & & 1 & $0.531^{* *}$ & $0.806^{* *}$ & $0.657^{* *}$ & $0.674^{* *}$ & $0.433^{* *}$ & $0.391^{*}$ & $0.408^{\star *}$ & $0.434^{* *}$ & $0.108^{* *}$ & $0.463^{* *}$ & $0.551^{* *}$ & $0.357^{* *}$ \\
\hline$C D$ & & & & & & 1 & $0.678^{* *}$ & $0.602^{* *}$ & $0.595^{\star \star}$ & $0.511^{* *}$ & $0.483^{* *}$ & $0.532^{* *}$ & $0.541^{\star *}$ & $0.341^{* *}$ & $0.433^{* *}$ & $0.510^{* *}$ & $0.401^{* *}$ \\
\hline $\mathrm{CC}$ & & & & & & & 1 & $0.678^{\star *}$ & $0.725^{\star \star}$ & $0.515^{\star \star}$ & $0.513^{\star *}$ & $0.488^{\star *}$ & $0.543^{\star \star}$ & $0.410^{* *}$ & $0.494^{\star \star}$ & $0.631^{\star *}$ & $0.359^{\star \star}$ \\
\hline FC & & & & & & & & 1 & $0.786^{\star \star}$ & $0.619^{* *}$ & $0.591^{* *}$ & $0.589^{\star *}$ & $0.610^{\star *}$ & $0.405^{\star \star}$ & $0.622^{*}$ & $0.667^{\star *}$ & $0.406^{\star *}$ \\
\hline $\mathrm{BC}$ & & & & & & & & & 1 & $0.597^{\star *}$ & $0.673^{\star *}$ & $0.551^{\star *}$ & $0.501^{\star *}$ & $0.437^{\star \star}$ & $0.538^{\star *}$ & $0.711^{* *}$ & $0.408^{* *}$ \\
\hline $\mathrm{HL}$ & & & & & & & & & & 1 & $0.699^{* *}$ & $0.463^{\star *}$ & $0.413^{\star *}$ & $0.417^{\star \star}$ & $0.446^{* *}$ & $0.533^{* *}$ & $0.359^{*}$ \\
\hline $\mathrm{FL}$ & & & & & & & & & & & 1 & $0.418^{\star *}$ & $0.484^{\star *}$ & $0.416^{* *}$ & $0.491^{* *}$ & $0.480^{* *}$ & $0.239^{*}$ \\
\hline EL & & & & & & & & & & & & 1 & $0.645^{\star \star}$ & $0.458^{* *}$ & $0.385^{\star *}$ & $0.401^{* *}$ & $0.412^{* *}$ \\
\hline EW & & & & & & & & & & & & & 1 & $0.435^{\star *}$ & $0.520^{\star *}$ & $0.398^{\star *}$ & $0.394^{\star *}$ \\
\hline EAI & & & & & & & & & & & & & & 1 & $0.322^{* *}$ & $0.404^{* *}$ & $0.304^{\star \star}$ \\
\hline EYI & & & & & & & & & & & & & & & 1 & $0.604^{\star *}$ & $0.241^{*}$ \\
\hline MC & & & & & & & & & & & & & & & & 1 & $0.348^{* *}$ \\
\hline TL & & & & & & & & & & & & & & & & & 1 \\
\hline
\end{tabular}

${ }^{*} P<0.05 ;{ }^{* *} P<0.01$

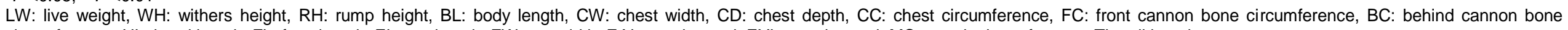
circumference, HL: head length, FL: face length, EL: ear length, EW: ear width, EAl: ears interval, EYI: eyes interval, MC: mouth circumference, TL: tail length 
Appendix Table 2 Estimates of phenotypic correlations among age and body dimensions in Zerdava dogs

\begin{tabular}{|c|c|c|c|c|c|c|c|c|c|c|c|c|c|c|c|c|c|c|c|}
\hline & Age & WH & $\mathrm{RH}$ & $\mathrm{BL}$ & $\mathrm{CW}$ & $C D$ & $\mathrm{CC}$ & FC & $\mathrm{BC}$ & LW & TL & TW & $\mathrm{HL}$ & FL & $\mathrm{EL}$ & EW & EAI & EYI & MC \\
\hline Age & 1 & $0.242^{*}$ & $0.349^{\star \star}$ & 0.172 & $0.320^{* *}$ & $0.543^{* *}$ & $0.457^{\star *}$ & $0.270^{\star *}$ & $0.226^{*}$ & $0.410^{\star *}$ & 0.134 & 0.128 & $0.263^{* *}$ & 0.072 & 0.06 & 0.167 & 0.175 & 0.189 & $0.221^{*}$ \\
\hline WH & & 1 & $0.835^{\star \star}$ & $0.487^{* *}$ & 0.142 & $0.577^{* *}$ & $0.540^{\star *}$ & $0.429^{\star *}$ & $0.385^{\star *}$ & $0.496^{\star *}$ & $0.375^{\star *}$ & $0.405^{\star *}$ & $0.567^{\star *}$ & $0.517^{\star \star}$ & $0.447^{* *}$ & $0.324^{* *}$ & 0.027 & $0.253^{*}$ & $0.393^{* *}$ \\
\hline $\mathrm{RH}$ & & & 1 & $0.490^{* *}$ & $0.208^{*}$ & $0.539^{\star *}$ & $0.556^{* *}$ & $0.460^{* *}$ & $0.410^{* *}$ & $0.444^{* *}$ & $0.317^{\star *}$ & $0.379^{* *}$ & $0.549^{* *}$ & $0.426^{\star *}$ & $0.454^{\star *}$ & $0.305^{\star *}$ & 0.015 & $0.219^{*}$ & $0.466^{\star *}$ \\
\hline $\mathrm{BL}$ & & & & 1 & 0.17 & $0.605^{\star *}$ & $0.554^{* *}$ & $0.564^{* *}$ & $0.471^{* *}$ & $0.343^{* *}$ & $0.197^{\star}$ & $0.431^{* *}$ & $0.418^{\star *}$ & $0.308^{* *}$ & $0.467^{* *}$ & $0.308^{* *}$ & -0.045 & 0.183 & $0.522^{\star *}$ \\
\hline $\mathrm{CW}$ & & & & & 1 & $0.344^{\star *}$ & $0.417^{* *}$ & $0.379^{* *}$ & $0.423^{\star *}$ & $0.461^{* *}$ & -0.058 & $0.274^{* *}$ & $0.238^{*}$ & $0.227^{\star}$ & $0.224^{*}$ & 0.163 & 0.099 & 0.028 & $0.325^{\star *}$ \\
\hline$C D$ & & & & & & 1 & $0.798^{* *}$ & $0.571^{* *}$ & $0.592^{* *}$ & $0.557^{\star \star}$ & $0.309^{* \star}$ & $0.424^{\star *}$ & $0.526^{* *}$ & $0.291^{* *}$ & $0.352^{\star *}$ & $0.314^{\star *}$ & 0.129 & 0.19 & $0.458^{* *}$ \\
\hline $\mathrm{CC}$ & & & & & & & 1 & $0.531^{* *}$ & $0.515^{\star \star}$ & $0.590^{\star *}$ & $0.335^{\star *}$ & $0.476^{* *}$ & $0.517^{* *}$ & $0.421^{* *}$ & $0.313^{* *}$ & 0.193 & 0.029 & 0.183 & $0.340^{* *}$ \\
\hline FC & & & & & & & & 1 & $0.773^{\star *}$ & $0.468^{\star *}$ & $0.337^{\star *}$ & $0.249^{*}$ & $0.559^{* *}$ & $0.393^{\star *}$ & $0.370^{* *}$ & $0.259^{* *}$ & $0.241^{*}$ & 0.151 & $0.537^{\star \star}$ \\
\hline $\mathrm{BC}$ & & & & & & & & & 1 & $0.475^{\star *}$ & $0.315^{\star *}$ & $0.322^{* *}$ & $0.572^{* *}$ & $0.392^{* *}$ & $0.425^{\star *}$ & $0.294^{* *}$ & 0.181 & 0.087 & $0.530^{\star \star}$ \\
\hline LW & & & & & & & & & & 1 & $0.242^{*}$ & $0.382^{* *}$ & $0.485^{\star *}$ & $0.438^{* *}$ & $0.349^{* *}$ & $0.256^{*}$ & -0.018 & $0.241^{*}$ & $0.333^{\star *}$ \\
\hline $\mathrm{TL}$ & & & & & & & & & & & 1 & $0.305^{\star \star}$ & $0.453^{\star *}$ & $0.272^{\star *}$ & 0.126 & 0.025 & 0.126 & $0.317^{\star \star}$ & 0.121 \\
\hline TW & & & & & & & & & & & & 1 & $0.322^{\star *}$ & $0.331^{* *}$ & 0.142 & $0.267^{\star \star}$ & -0.035 & $0.388^{\star *}$ & 0.137 \\
\hline $\mathrm{HL}$ & & & & & & & & & & & & & 1 & $0.611^{* *}$ & $0.433^{\star *}$ & $0.276^{\star *}$ & $0.301^{* *}$ & $0.345^{\star \star}$ & $0.469^{* *}$ \\
\hline $\mathrm{FL}$ & & & & & & & & & & & & & & 1 & $0.406^{* *}$ & $0.268^{* *}$ & 0.042 & $0.213^{*}$ & $0.335^{\star *}$ \\
\hline EL & & & & & & & & & & & & & & & 1 & $0.289^{\star *}$ & 0.047 & -0.02 & $0.473^{\star \star}$ \\
\hline EW & & & & & & & & & & & & & & & & 1 & 0.1 & $0.213^{*}$ & $0.411^{\star *}$ \\
\hline EAI & & & & & & & & & & & & & & & & & 1 & -0.033 & 0.111 \\
\hline EYI & & & & & & & & & & & & & & & & & & 1 & 0.074 \\
\hline MC & & & & & & & & & & & & & & & & & & & 1 \\
\hline
\end{tabular}

${ }^{\star} P<0.05,{ }^{* *} P<0.01$



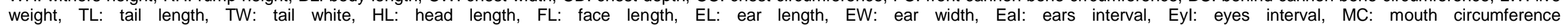


Appendix Table 3 Estimates of phenotypic correlations among age and body dimensions in Çatalburun dogs

\begin{tabular}{|c|c|c|c|c|c|c|c|c|c|c|c|c|c|c|c|c|c|}
\hline & Age & WH & $\mathrm{RH}$ & $\mathrm{BL}$ & CW & $C D$ & $\mathrm{CC}$ & FC & $\mathrm{BC}$ & LW & $\mathrm{HL}$ & $\mathrm{FL}$ & $\mathrm{EL}$ & EW & EAI & EYI & MC \\
\hline Age & 1 & $0.264^{* *}$ & $0.243^{\star}$ & $0.232^{*}$ & $0.236^{*}$ & $0.262^{* *}$ & $0.422^{\star *}$ & $0.460^{* *}$ & $0.419^{\star *}$ & $0.317^{* *}$ & $0.325^{\star *}$ & $0.386^{* *}$ & $0.249^{*}$ & $0.283^{* *}$ & 0,008 & 0,145 & $0.276^{* *}$ \\
\hline WH & & 1 & $0.878^{* *}$ & $0.616^{* *}$ & $0.363^{\star *}$ & $0.485^{\star *}$ & $0.718^{* *}$ & $0.675^{\star *}$ & $0.502^{\star *}$ & $0.644^{* *}$ & $0.586^{\star *}$ & $0.633^{* *}$ & $0.443^{* *}$ & $0.432^{* *}$ & $0.199^{*}$ & $0.225^{\star}$ & $0.634^{* *}$ \\
\hline $\mathrm{RH}$ & & & 1 & $0.465^{\star *}$ & $0.381^{* *}$ & $0.545^{\star *}$ & $0.738^{* *}$ & $0.709^{* *}$ & $0.581^{* *}$ & $0.653^{* *}$ & $0.628^{\star *}$ & $0.613^{* *}$ & $0.521^{* *}$ & $0.526^{* *}$ & $0.289^{* *}$ & $0.255^{\star}$ & $0.627^{* *}$ \\
\hline $\mathrm{BL}$ & & & & 1 & $0.326^{* *}$ & $0.542^{* *}$ & $0.602^{* *}$ & $0.451^{* *}$ & $0.346^{\star *}$ & $0.421^{\star *}$ & $0.423^{* *}$ & $0.489^{* *}$ & $0.267^{* *}$ & $0.337^{* *}$ & $0.357^{\star *}$ & 0,152 & $0.494^{* *}$ \\
\hline $\mathrm{CW}$ & & & & & 1 & $0.398^{* *}$ & $0.554^{\star *}$ & $0.537^{\star *}$ & $0.389^{* *}$ & $0.501^{* *}$ & $0.454^{\star *}$ & $0.425^{* *}$ & $0.444^{* *}$ & $0.364^{* *}$ & $0.207^{*}$ & $0.212^{*}$ & $0.460^{* *}$ \\
\hline$C D$ & & & & & & 1 & $0.714^{\star *}$ & $0.505^{\star *}$ & $0.434^{\star *}$ & $0.571^{* *}$ & $0.480^{\star *}$ & $0.476^{* *}$ & $0.375^{\star *}$ & $0.398^{* *}$ & $0.400^{\star *}$ & 0,179 & $0.477^{\star *}$ \\
\hline $\mathrm{CC}$ & & & & & & & 1 & $0.681^{* *}$ & $0.563^{\star *}$ & $0.822^{* *}$ & $0.603^{* *}$ & $0.640^{* *}$ & $0.525^{\star *}$ & $0.500^{* *}$ & $0.416^{\star *}$ & 0,147 & $0.655^{\star *}$ \\
\hline $\mathrm{FC}$ & & & & & & & & 1 & $0.806^{\star *}$ & $0.625^{\star *}$ & $0.641^{* *}$ & $0.640^{* *}$ & $0.628^{* *}$ & $0.595^{\star \star}$ & $0.321^{\star *}$ & $0.349^{* *}$ & $0.657^{\star \star}$ \\
\hline $\mathrm{BC}$ & & & & & & & & & 1 & $0.531^{\star *}$ & $0.550^{* *}$ & $0.532^{* *}$ & $0.509^{* \star}$ & $0.636^{* *}$ & $0.353^{\star *}$ & $0.252^{*}$ & $0.551^{* *}$ \\
\hline LW & & & & & & & & & & 1 & $0.553^{* *}$ & $0.600^{* *}$ & $0.452^{\star *}$ & $0.410^{* *}$ & $0.319^{* *}$ & 0,079 & $0.555^{\star \star}$ \\
\hline $\mathrm{HL}$ & & & & & & & & & & & 1 & $0.756^{* *}$ & $0.558^{* *}$ & $0.562^{* *}$ & $0.341^{* *}$ & $0.296^{\star *}$ & $0.609^{* *}$ \\
\hline $\mathrm{FL}$ & & & & & & & & & & & & 1 & $0.545^{\star *}$ & $0.527^{* *}$ & $0.363^{* *}$ & $0.299^{\star *}$ & $0.575^{\star \star}$ \\
\hline EL & & & & & & & & & & & & & 1 & $0.538^{\star *}$ & $0.207^{*}$ & $0.397^{\star \star}$ & $0.537^{\star \star}$ \\
\hline EW & & & & & & & & & & & & & & 1 & $0.398^{\star *}$ & $0.317^{\star *}$ & $0.418^{\star *}$ \\
\hline EAl & & & & & & & & & & & & & & & 1 & 0,038 & $0.249^{*}$ \\
\hline $\mathrm{EYI}$ & & & & & & & & & & & & & & & & 1 & $0.403^{\star \star}$ \\
\hline $\mathrm{MC}$ & & & & & & & & & & & & & & & & & 1 \\
\hline
\end{tabular}

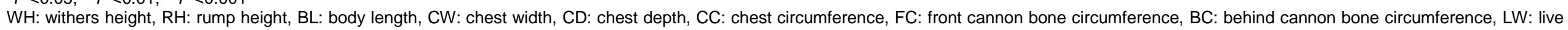
weight, $\mathrm{HL}$ : head length, FL: face length, EL: ear length, EW: ear width, EAl: ears interval, EYI: eyes interval, MC: mouth circumference 